\title{
Asociación del momento de administración de dexametasona intratimpánica con los resultados auditivos en pacientes con hipoacusia súbita idiopática: 101 casos
}

\author{
Association of the moment of administration of intratympanic dexamethasone with the \\ auditory results in patients with sudden idiopathic hearing loss: 101 cases
}

Luis R. Maldonado-Sánchez ${ }^{1,2 *}$ y Fernando Pineda-Cásarez ${ }^{1}$

${ }^{1}$ Departamento de Otorrinolaringología y Cirugía de Cabeza y Cuello, Hospital Regional General Ignacio Zaragoza, Instituto de Seguridad y Servicios Sociales de los Trabajadores del Estado; '²Facultad Mexicana de Medicina, Universidad La Salle de México. Ciudad de México, México

\section{Resumen}

Objetivo: Evaluar la asociación del momento de administración de dexametasona intratimpánica con los resultados audititivos en pacientes con hipoacusia súbita idiopática. Método: Estudio observacional, longitudinal, analítico y retrospectivo dê pacientes tratados por hipoacusia súbita idiopática con fosfato sódico de dexametasona como manejo inicial. Se compararôn el umbral auditivo, la ganancia auditiva $(d B)$ y el porcentaje de pacientes que mejoraron de los que iniciaron tratamiento en la primera semana (grupo 1S) y aquellos que lo iniciaron a partir de la segunda semana (grupo 2S). Se consideró significátivo un valor de $p$ < 0.05. Resultados: Se incluyeron 101 pacientes (37 hombres y 64 mujeres). Antes de la enfermedad: los pacientes tenían una función auditiva similar. Tras el tratamiento, los pacientes del grupo $1 \mathrm{~S}$ tuvieron significativamente mejor discriminación fonética que los del grupo $2 S$ (33.9 $\pm 18.3 \mathrm{vs}$. $43.6 \pm 26.1 \mathrm{~dB} ; p=0.036)$. La ganancia auditiva en el grupo $1 S$ fue de $32.0 \pm 11.9 \mathrm{~dB}$ y en el grupo $2 S$ fue de $18.2 \pm 9.5 \mathrm{~dB}(p<0.001)$. El 93.5\% de los que iniciaron tratamiento la primera semana tuvieron mejora auditiva y el $61.8 \%$ en la segunda semana $(p<0.001)$. Conclusiones: El fosfato sódico de dexametasona intratimpánico administrado en la primera semana, en comparación con la segunda semana, fue más eficaz e indujo mejor discriminación fonética en la mayoría de los casos.

Palabras clave: Dexametasona. Hipoacusia súbita idiopática. Resultados.

\begin{abstract}
Objective: To evaluate the association of the moment of administration of intratympanic dexamethasone with the auditory results in patients with sudden idiopathic hearing loss. Method: An observational, longitudinal, analytical, retrospective study on patients treated for sudden idiopathic hearing loss with dexamethasone sodium phosphate as initial management. Auditory threshold, auditory gain $(\mathrm{dB})$ and percentage of patients who improved from those who started treatment in the first week (group 1S) versus those started management from the second week (group 2S) are compared. A $p<0.05$ was considered significant. Results: 101 cases (37 males and 64 female) were included. Before disease, patients had similar auditory function, after treatment patients in group $1 S$ had significantly better phonetic discrimination than those in group $2 S$ (33.9 $\pm 18, \overline{\overline{3}}$ vs. $43.6 \pm 26.1 \mathrm{~dB} ; p=0.036)$ hearing gain in group $1 S$ was $32.0 \pm 11.9 \mathrm{~dB}$ and in group $2 S$ was $18.2 \pm 9.5 \mathrm{~dB}(p<0.001) .93,5 \%$

\section{Correspondencia:}

*Luis R. Maldonado-Sánchez

Calle E, Lote 9, Manzana 16

Col. Citlalmina

Fecha de recepción: 09-01-2020

C.P. 56540, Ixtapaluca, Edo. de México, México

E-mail: dr.rafamaldonado@ hotmail.com

Cir Cir. 2021;89(1):77-82

Contents available at Pugimed www.cirugiaycirujanos.com
la licencia CC BY-NC-ND (http://creativecommons.org/licenses/by-nc-nd/4.0/).
\end{abstract}


of those who started treatment the first week had auditory improvement while only $61.8 \%$ in second week $(p<0.001)$. Conclusions: Intratympanic dexamethasone sodium phosphate administered in the first week was more effective in indüing better phonetic discrimination in most cases and is safe than in the second week.

Key words: Dexamethasone. Sudden idiopathic hearing loss. Results.

\section{Introducción}

La hipoacusia súbita idiopática se caracteriza por una pérdida sensorineural de más de $30 \mathrm{~dB}$ en tres o más frecuencias puras consecutivas, establecida en menos de 3 días, habiendo descartado todas las etiologías de hipoacusia súbita ${ }^{1,2}$. Esta condición se considera una emergencia otorrinolaringológica poco común, con una incidencia de $5-20$ casos por 100,000

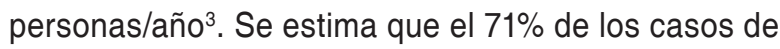
pérdida auditiva aguda son idiopáticos ${ }^{4}$.

Aunque su fisiopatología no es clara, se han propuesto diversas causas infecciosas, vasculares e inmunitarias $^{5,6}$. El oído interno es particularmente sensible a la isquemia transitoria debido a sus altos requerimientos metabólicos y a la falta de aporte vascular colateral ${ }^{7}$. Los estudios histopatológicos han encontrado una pérdida de células ciliadas y células de soporte del órgano de Corti, pérdida de la membrana tectorial, células de soporte y estría vascular, pérdida de neuronas cocleares y alteraciones vasculares, entre otras, que podrían resultar de la activación patológica de las vías de estrés celular y de inflamación que involucran el factor nuclear kappa $B$ dentro de la cóclea, sin datos histopatológicos que sugieran roturas de membrana, fístulas perilinfáticas u oclusión vascular, invasión leucocitaria, hipervascularidad o hemorragia dentro del laberinto, como podría esperarse en la cocleítis viral ${ }^{8}$.

Una variedad de factores que son conocidos afecta la recuperación de la hipoacusia sensorineural, incluyendo la duración de la enfermedad, la gravedad de la pérdida auditiva, la edad y la presencia de otros síntomas ${ }^{9}$.

Durante el abordaje diagnóstico es necesaria una valoración clínica y paraclínica cuidadosa para excluir las causas que pueden ser mortales o tratables, como eventos vasculares y enfermedades malignas, y además, los pacientes deben ser referidos con urgencia a evaluaciones adicionales que incluyan estudios de imagen y serológicos ${ }^{10}$.

Entre las estrategias terapéuticas utilizadas hasta el momento se encuentran antivirales, agentes vasoactivos a base de oxígeno y corticosteroides ${ }^{11,12}$. Sin embargo, los corticosteroides son los únicos compuestos útiles para el manejo de la hipoacuisia súbita crónica, y el mecanismo de acción propuejsto es su acción inflamatoria y antiestrés celular, quě ha demostrado disminuir la inflamación y el edema el el oído interno ${ }^{13}$. Estudios previos han demostrado que la aplicación intratimpánica de corticosteroidesu es igual de efectiva que la prednisona oral y se asocia, por tanto, con menores efectos adversos que las dosis altas de corticosteroides orales ${ }^{14-16}$. Sin embarigo, de acuerdo con reportes previos, es diez veces más común el uso de corticosteroides por vía oral, y $\frac{\subseteq}{\text { solo }}$ el $8 \%$ de los otorrinolaringólogos en los Estados छ̈nidos de América utilizan corticosteroides intratimpánicos, lo que condiciona que los reportes sobre el u्uso intratimpánico sean escasos y en su mayoría con pequeños tamaños de muestra ${ }^{13}$.

En el presente estudio se evalúa la asociación del momento de administración de dexametasona intratimpánica con los resultados auditivos en pacientes con hipoacusia súbita idiopática que previamente no habían recibido tratamiento.

\section{Método}

Se realizó un estudio observacional, longitudinal, analítico y retrospectivo de casos de pacientes que fueron atendidos en el Hospital Regional GeneraPlgnacio Zaragoza, en Ciudad de México, por hipoactisia súbita idiopática en el periodo 2009-2019. Se incluyeron pacientes sin tratamiento previo y que presentaran una pérdida auditiva sensorineural de $30 \mathrm{~d} \overline{\mathrm{B}}$ en tres frecuencias consecutivas en menos de 72 horas, con audición normal en el oído contralateral y otoscopia normal. Se protocolizaron realizando hemoğgama completo, perfil metabólico, electrolitos séricos y velocidad de sedimentación globular normales; ešstudio diagnóstico reumatológico incluyendo anticuerpos antinucleares, que se indicaron cuando la historia fue sugestiva de etiología autoinmunitaria; VDRL (Vènereal Disease Research Laboratory) y prueba fluorescente de anticuerpos treponémicos si la sospechá de neurosífilis era alta; y hallazgos normales en la tomografía computada contrastada de cortes finos en 
cráneo y conductos auditivos internos. En caso de sospecha de neoplasia en el oído interno basada en los resultados de la tomografía, se les realizó también resonancia magnética con gadolinio de cerebro y de canales auditivos internos; el único paciente que presentó neoplasia fue descartado para este estudio. No se incluyeron pacientes alérgicos a la dexametasona ni con antecedentes de otitis media crónica, trauma de cráneo o acústico, enfermedad de Ménière, meningitis, cirugía otológica, radiación, exposición a medicamentos ototóxicos o perforación timpánica homolateral. Se eliminaron los pacientes que no completaron el tratamiento con dexametasona intratimpánica, así como aquellos que presentaron alergia a la dexametasona u otitis externa o media aguda.

La audiometría de tonos puros se realizó previamente al tratamiento y un mes después de finalizarlo, por el mismo audiólogo profesional y con un equipo calibrado bajo la norma para auriculares y transductores óseos. Los umbrales auditivos con estímulos de vía aérea y conductiva en ambos oídos fueron establecidos en frecuencias a 250, 500, 1000, 2000, 4000,6000 y $8000 \mathrm{~Hz}$, agregando $3000 \mathrm{~Hz}$ si se encontraba una diferencia de $20 \mathrm{~dB}$ entre los umbrales de 2000 y $4000 \mathrm{~Hz}$. Se determinó discapacidad auditiva con el promedio de tonos puros en los umbrales auditivos a 500, 1000, 2000 y $4000 \mathrm{~Hz}$. Un umbral auditivo de hasta $24 \mathrm{~dB}$ fue definido como normal, un umbral de 25-39 dB como hipoacusia superficial, un umbral de 40-59 dB como hipoacusia moderada, un umbral de 60-79 dB como hipoacusia grave, un umbral de 80-99 dB como hipoacusia profunda, y una pérdida $>100 \mathrm{~dB}$ se definió como anacusia ${ }^{17}$.

Se obtuvo información clínica relevante de los expedientes, incluyendo edad, sexo, oído afectado, tiempo desde el inicio de los síntomas hasta el tratamiento y comorbilidad, como sobrepeso, diabetes meIlitus, dislipidemia e hipotiroidismo.

Se consideró mejoría auditiva una ganancia en tonos puros $>20 \mathrm{~dB}$ o una discriminación fonética $>20 \%$ un mes posterior al término de la terapia intratimpánica.

El tratamiento se realizó en todos los casos en el consultorio, con el paciente en decúbito contralateral al oído afectado y con la cabeza girada 45 grados, y consistió en la aplicación de cinco dosis $(2 \mathrm{mg} / 0.5 \mathrm{ml})$ de fosfato sódico de dexametasona, $2 \mathrm{mg}$, con una separación de 24 horas. Primero se infiltran $10 \mathrm{mg}$ de lidocaína al $2 \%$ en el conducto auditivo externo, en su tercio medio, bajo visión microscópica (OPMI pico
S100, Carl Zeiss, Oberkochen, Alemania), y con una aguja $24 \mathrm{G}$ se realiza una punción en el cuadrante posteroinferior de la membrana timpánica para administrar la dexametasona. Se mantiene en posición al paciente por 30 minutos, tiempo óptimo para la diffusión hacia el oído interno a través de la membrăna redonda. Previo a la colocación del fármaco se pide al paciente que evite deglutir, hablar o realizar maniobras de Valsalva para impedir la eliminación del iomedicamento a través de la trompa de Eustaquio, ¿y al egresar del consultorio se le indica que realice cuidado del oído seco en el oído en tratamiento, colocan̆do una bola de algodón y cubriendo la superficie extêrna con vaselina sólida hasta que cicatrice la membrana timpánica, lo cual sucede en el primer mes. Al administrar el medicamento, el $22.7 \%$ presentaron vértigo que remitió en los primeros 10 minutos, y el $6.9 \%$ notaron otodinia ardorosa leve.

Este estudio fue aprobado por el Comité Locał de Bioética e Investigación, y tomó en cuenta las consideraciones éticas para investigación en seres humanos de la Declaración de Helsinki, del Códigö de Nuremberg y de la Ley General en Salud de los Estados Unidos Mexicanos.

El análisis estadístico se realizó con el progrăma SPSS v.25, con el cual se realizaron pruebas estadísticas descriptivas consistentes en media y desviación estándar para las variables cuantitativas, y frecuencias y porcentajes para las variables cualitativas? $\mathrm{Se}$ utilizaron las pruebas de ji al cuadrado y exacta- de Fisher para determinar diferencias significativas en las variables cualitativas, y las pruebas $t$ de Stu $\overline{\text { dent }}$ o $t$ de muestras relacionadas para las variabiles cuantitativas. Se consideró significativo un valoẹ de $p<0.05$.

\section{Resultados}

Se incluyeron 101 pacientes que cumplieron criterios de hipoacusia súbita idiopática y que fuëron tratados con fosfato sódico de dexametasona intratimpánica, con una edad promedio de $50.0=5.3$ años. Dos terceras partes eran mujeres y una tercera parte eran varones. El $63.3 \%$ tenían alguna comorbilidad. Las principales enfermedades concomitantes fueron dislipidemias (33.7\%), hipertensión (25.7\%) y diabetes mellitus (17.8\%). El oído afectado fue ef izquierdo en el $55.4 \%$ de los casos y el derecho en el $44.6 \%$ (Tabla 1). Un paciente fue diagnosticado neurosífilis y se excluyó del estudio. 
Tabla 1. Características generales de los pacientes incluidos ( $n=101)$

\begin{tabular}{lc}
\hline Edad (años) & $50.0 \pm 15.3$ \\
Sexo, \% (n) & \\
$\quad$ Masculino & $36.6(37)$ \\
Femenino & $63.4(64)$ \\
Comorbilidad, \% (n) & \\
Diabetes mellitus & $17.8(18)$ \\
Hipertensión & $25.7(26)$ \\
Dislipidemia & $33.7(34)$ \\
Sobrepeso/obesidad & $15.8(16)$ \\
Enfermedad renal crónica & $4.0(4)$ \\
Hipotiroidismo & $2.0(2)$ \\
Oído afectado, \% (n) & \\
Izquierdo & $55.4(56)$ \\
Derecho & $44.6(45)$ \\
\hline
\end{tabular}

Tabla 2. Comparación de eventos adversos del tratamiento entre grupos

\begin{tabular}{lccc}
\hline $\begin{array}{l}\text { Eventos } \\
\text { adversos }\end{array}$ & $\begin{array}{c}\text { Grupo } \mathbf{1 S}(\mathbf{n}=\mathbf{4 6}) \\
\%(\mathbf{n})\end{array}$ & $\begin{array}{c}\text { Grupo } \mathbf{2 S}(\mathbf{n}=\mathbf{5 5}) \\
\%(\mathbf{n})\end{array}$ & $\mathbf{p}$ \\
\hline Otodinia & $2.1(1)$ & $10.9(6)$ & \\
Vértigo & $19.6(9)$ & $25.5(14)$ & 0.142 \\
Ninguno & $78.3(36)$ & $63.6(35)$ & \\
\hline
\end{tabular}

Un total de 46 pacientes iniciaron el tratamiento durante la primera semana posterior al inicio del cuadro clínico (grupo S1) y 55 pacientes lo iniciaron a las 2 semanas (grupo S2). Previo al tratamiento no hubo diferencias significativas en el umbral auditivo entre grupos. Sin embargo, tras el tratamiento, los pacientes del grupo S1 tuvieron significativamente mejor discriminación fonética que los del grupo S2 (Fig. 1). En consecuencia, tras el tratamiento los pacientes del grupo S1 tuvieron significativamente mayor ganancia auditiva que los del grupo S2 $(32.0 \pm 11.9$ vs. $18.2 \pm 9.5 \mathrm{~dB} ; p<0.001)$ (Fig. 2). Al comparar el porcentaje de pacientes que tuvieron mejoría auditiva tras el tratamiento, el $93.5 \%$ de los que iniciaron el tratamiento a la primera semana mejoraron y el $61.8 \%$ de los que iniciaron en la segunda semana $(p<0.001)$ (Fig. 3). No se encontraron diferencias significativas entre grupos en la frecuencia de eventos adversos durante la aplicación (Tabla 2).

En los pacientes con pérdida auditiva inicial superficial, moderada y grave, la tasa de mejora fue del 81.8-88.9\%, en comparación con el 53.3-57.1\% en los pacientes que al inicio presentaban una pérdida auditiva profunda 0 anacusia $(p=0.033$ ) (Tabla 3 ).

El $63.3 \%$ de los pacientes presentaban alguna comorbilidad. La diferencia entre los pacientes con y sin

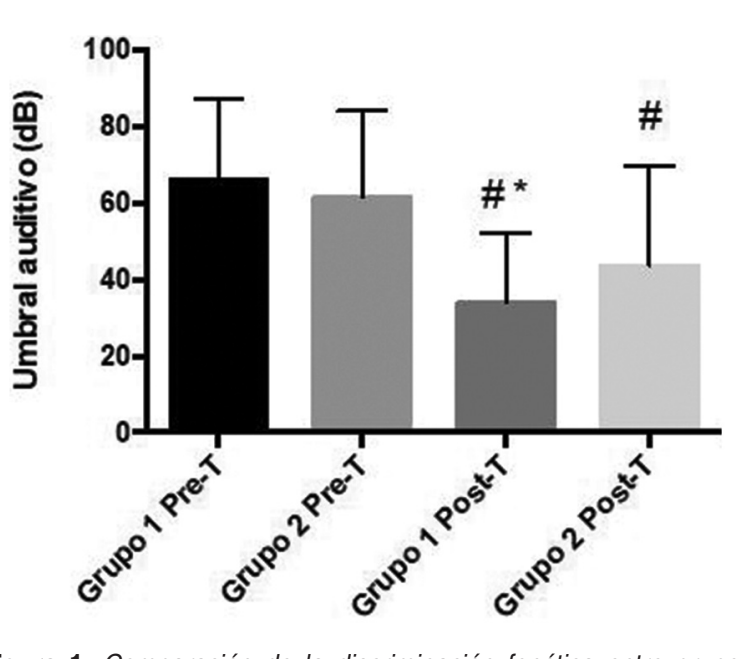

Figura 1. Comparación de la discriminación fonética entre grupos antes y después del tratamiento, y decibelios de ganancia. $p<0.001$. *Prueba t de muestras independientes vs. grupo 2S. "Prueba t pareada vs. antes del tratamiento.

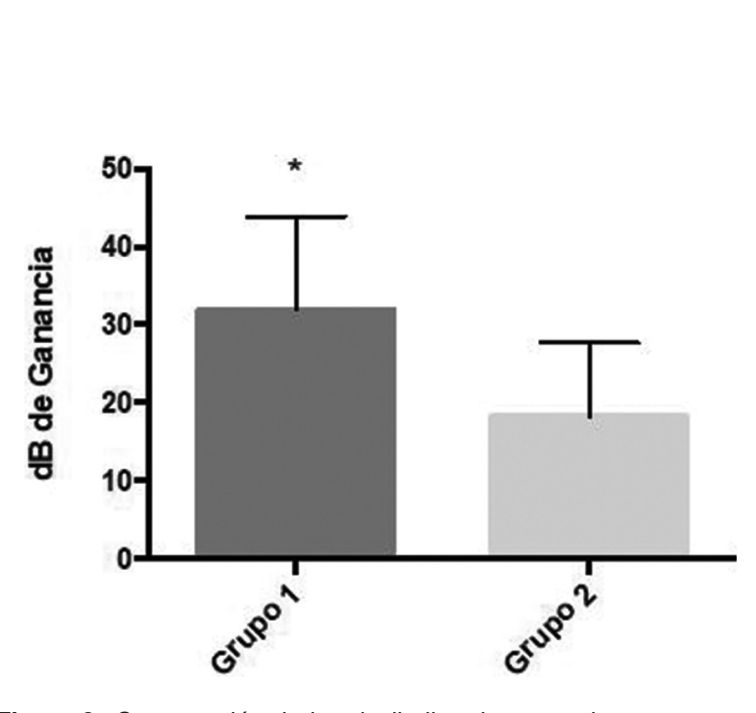

Figura 2. Comparación de los decibelios de ganancia entre grupos. $p<0.001$. *Prueba t de Student.

comorbilidad que mejoraron, $75 \%$ (48) vs. $78.3 \%$ (29), no fue estadísticamente significativa $(p=0.700)$ (Tabla 4).

\section{Discusión}

En este estudio se presentan los resultados del manejo de la hipoacusia súbita idiopática de unīperiodo de 11 años, comparando los resultados logrados según el tiempo transcurrido desde el inicio del êuadro clínico hasta el tratamiento. Encontramos que el tratamiento con fosfato sódico de dexametasona mejoró la discriminación fonética en ambos grupos, pero esta ganancia fue mayor en quienes iniciaron el 


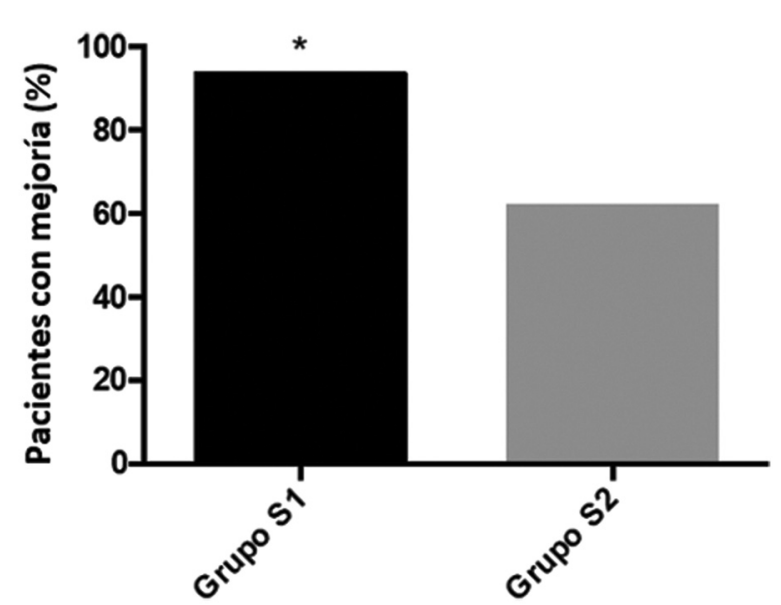

Figura 3. Porcentaje de pacientes con mejoría. *Prueba de Ji al cuadrado vs. grupo $2 S$.

Tabla 3. Porcentaje de pacientes que mejoraron según la categoría inicial de pérdida auditiva

\begin{tabular}{lccc}
\hline Pérdida auditiva inicial & $\begin{array}{c}\text { Mejoría } \\
(\mathbf{n}=\mathbf{7 7}) \%(\mathbf{n})\end{array}$ & $\begin{array}{c}\text { No mejoría } \\
(\mathbf{n}=\mathbf{2 4}) \%(\mathbf{n})\end{array}$ & $\mathbf{p}$ \\
\hline Superficial & $88.9(16)$ & $11.1(2)$ & 0.033 \\
Moderada & $81.8(27)$ & $18.2(6)$ & \\
Grave & $85.7(18)$ & $14.3(3)$ & \\
Profunda & $53.3(8)$ & $46.7(7)$ & \\
Anacusia & $57.1(8)$ & $42.9(6)$ & \\
\hline
\end{tabular}

Tabla 4. Respuesta al tratamiento en pacientes con y sin comorbilidad

\begin{tabular}{lccc}
\hline Resultados & $\begin{array}{c}\text { Sin comorbilidad } \\
(\mathbf{n}=\mathbf{3 7}) \%(\mathbf{n})\end{array}$ & $\begin{array}{c}\text { Con comorbilidad } \\
(\mathbf{n}=\mathbf{6 4}) \%(\mathbf{n})\end{array}$ & $\mathbf{p}$ \\
\hline Mejoría & $78.3(29)$ & $75(48)$ & 0.700 \\
No mejoría & $21.6(8)$ & $25(16)$ & \\
\hline
\end{tabular}

tratamiento en la primera semana del inicio de los síntomas que en aquellos que iniciaron el tratamiento a partir de la segunda semana. Los resultados auditivos no fueron diferentes entre los pacientes con comorbilidad y los que no la presentaban. Estos hallazgos destacan la importancia de identificar la pérdida auditiva súbita e iniciar oportunamente el tratamiento, ya que los resultados son mejores con el inicio del tratamiento en la primera semana, de acuerdo con nuestros hallazgos.

En un estudio realizado por Narozny, et al. ${ }^{18}$ para identificar los factores pronósticos en pacientes con hipoacusia súbita idiopática se encontró que el retraso del tratamiento es un factor pronóstico importante, encontrando mejores resultados cuando el tratamiento comenzó dentro de los 10 días posteriores al iñicio de los síntomas, lo cual es similar a lo encontradó en nuestro estudio.

En otro estudio no se encontraron diferencias șignificativas en la tasa de recuperación en pacientes que iniciaron el tratamiento de forma temprana ostardía, aunque los que iniciaron el tratamiento tempranamente (en los primeros 5 días) tuvieron con mâyor frecuencia mejora auditiva ${ }^{19}$. En otro estudio, en 40 pacientes se encontró que los que respondieron a corticosteroides tuvieron significativamente un menor tiempo entre el inicio de los síntomas y la aplicación de dexametasona intratimpánica ${ }^{20}$. Sin embargo, el tiempo promedio desde el inicio de los síntomas hăsta el tratamiento fue mucho más largo (40 días en promedio) que en nuestra serie de casos. Quizá a êsto se deba, al menos en parte, que la tasa de recuperación de los pacientes fue de solo el $40 \%$ en la serie de casos de Haynes, et al. ${ }^{20}$, y superior al $90 \%$ en nuestros pacientes cuando iniciaron el tratamiento en la primera semana desde el inicio de los síntomás y del $61.8 \%$ en los que iniciaron el tratamiento èn la segunda semana.

Por su parte, Battista ${ }^{21}$, en una serie de 25 pacientes con hipoacusia súbita idiopática a los quę se aplicó dexametasona intratimpánica como tratamiènto inicial en los 28 días posteriores al inicio de los ${ }^{-}$íntomas, reportó unas tasas de mejoría total del $8 \%$ y de mejoría parcial del $12 \%$, cifras inferiores a laŝlogradas en nuestro estudio. Las principales diferencias con respecto a nuestra serie de casos son que su serie solo incluía pacientes con sordera profunda y con un promedio de 28 días desde el inicio de síntomas, y nosotros incluimos pacientes con distinitos grados de gravedad de la sordera y un buen número de pacientes que iniciaron la terapia durante la primera semana de inicio de los síntomas, lo que señala la importancia del inicio temprano del tratamiento y que el grado de mejoría está también en relación con la gravedad de la pérdida auditiva. De hecho, en nuestra serie, los pacientes con pérdida auditiva profun बa 0 anacusia inicial tuvieron menor tasa de mejoria gue aquellos con menor pérdida auditiva inicial.

Por otro lado, los corticosteroides intratimpánicos fueron seguros y se asociaron a una baja frecuegcia de efectos adversos, que incluyeron otodinia y vêrtigo, y que no ameritaron cuidados adicionales; nö se presentaron eventos adversos más graves. De heeho, se ha demostrado que, en comparación con losucorticosteroides sistémicos, el uso de corticosteroides 
intratimpánicos se asocia con menores efectos adversos, y dado que ambos tienen la misma efectividad se prefieren los intratimpánicos para evitar los efectos adversos de la administración sistémica ${ }^{22}$.

Si bien este estudio es de carácter observacional y tiene las debilidades inherentes a este tipo de estudios, su fortaleza estriba en que el tamaño de muestra supera los 100 casos y es uno de los pocos estudios reportados sobre el manejo inicial de la hipoacusia súbita idiopática con corticosteroides intratimpánicos, ya que la mayoría de los reportes existentes en la literatura son sobre el uso de corticosteroides como terapia de rescate en pacientes que no tuvieron respuesta inicial a otra modalidad de tratamiento ${ }^{20}$. La proporción de mejora auditiva fue más alta que en otros estudios.

\section{Conclusiones}

La aplicación intratimpánica de fosfato sódico de dexametasona en la primera semana posterior al inicio de los síntomas es efectiva, induce mejoría auditiva en la mayoría de los casos y es segura. Por lo tanto, se recomienda administrarla lo más temprano posible para maximizar las posibilidades de éxito en los pacientes con hipoacusia súbita idiopática.

\section{Financiamiento}

Los autores declaran que no se obtuvo ningún tipo de financiamiento por parte de ninguna empresa.

\section{Conflicto de intereses}

Los autores declaran que no existe ningún conflicto de intereses.

\section{Responsabilidades éticas}

Protección de personas y animales. Los autores declaran que para esta investigación no se han realizado experimentos en seres humanos ni en animales.

Confidencialidad de los datos. Los autores declaran que han seguido los protocolos de su centro de trabajo sobre la publicación de datos de pacientes.
Derecho a la privacidad y consentimiento informado. Los autores han obtenido el consentimiento informado de los pacientes y/o sujetos referidos en el artículo. Este documento obra en poder del autoóde correspondencia.

\section{Bibliografía}

1. Kuhn M, Heman-Ackah SE, Shaikh JA, Roehm PC. Sudden sensợineural hearing loss: a review of diagnosis, treatment, and prognosis. Trends Amplif. 2011;15:91-105.

2. Plaza G, Durio E, Herráiz C, Rivera T, García-Berrocal JR. Consenso sobre el diagnóstico y tratamiento de la sordera súbita. Acta Otorīnolaringol Esp. 2011;62:144-57.

3. Stachler RJ, Chandrasekhar SS, Archer SM, Rosenfeld RM, Schwartz SR, Barrs DM, et al. Clinical practice guideline: sudden hearing loss. Otolaryngol Head Neck Surg. 2012;146(1S):S1-S35.

4. Chau JK, Lin JRJ, Atashband S, Irvine RA, Westerberg BD. Systematic review of the evidence for the etiology of adult sudden sensorineural hearing loss. Laryngoscope. 2010;120:1011-21.

5. Merchant SN, Durand ML, Adams JC. Sudden deafness: is it viral?ORL J Otorhinolaryngol Relat Spec. 2008;70:52-62.

6. Kikidis D, Nikolopoulos TP, Kampessis G, Stamatiou G, Chrysovergis A. Sudden sensorineural hearing loss: subclinical viral and toxoplasmosis infections as aetiology and how they alter the clinical course. ORL. 2011;73:110-5.

7. Lin CF, Lee KJ, Yu SS, Lin YS. Effect of comorbid diabetes and h̆ypercholesterolemia on the prognosis of idiopathic sudden sensorineural hearing loss. Laryngoscope. 2016;126:142-9.

8. Merchant SN, Adams JC, Nadol JB. Pathology and pathophysiology of idiopathic sudden sensorineural hearing loss. Otol Neurotol. 2005;26:15j-60.

9. Chang J, Kang CJ, Yueh CY, Fang KH, Yeh RM, Tsai YT. The relationship between serum lipids and sudden sensorineural hearing $\overline{\bar{O} \mathbf{s} s} \mathrm{~s}$; systematic review and meta-analysis. PLoS One. 2015;10:e0121025.

10. Schreiber BE, Agrup C, Haskard DO, Luxon LM. Sudden sensoriheural hearing loss. Lancet. 2010;375:1203-11.

11. Wei BPC, Stathopoulos D, O'Leary S. Steroids for idiopathic sudden sensorineural hearing loss. Cochrane Database Syst Rev. 2013;(7):CD003998.

12. Isaacson B. Hearing loss. Med Clin North Am. 2010;94:973-88.

13. Lawrence R, Thevasagayam R. Controversies in the management of sudden sensorineural hearing loss: an evidence-based review. ClibOtolaryngol. 2015;40:176-82.

14. Rauch SD, Halpin CF, Antonelli PJ, Babu S, Carey JP, Gantz BJ,et al. Oral vs intratympanic corticosteroid therapy for idiopathic sudden sensorineural hearing loss: a randomized trial. JAMA. 2011;305:2071-9

15. Metrailer AM, Babu SC. Management of sudden sensorineural heáring loss. Curr Opin Otolaryngol Head Neck Surg. 2016;24:403-6.

16. Spear SA, Schwartz SR. Intratympanic steroids for sudden sensorineural hearing loss: a systematic review. Otolaryngol Head Neck Surg. 2011;145:534-43.

17. Vilayur E, Gopinath B, Harris DC, Burlutsky G, McMahon CM, Mitchell P. The association between reduced GFR and hearing loss: a cross-sectional population-based study. Am J Kidney Dis. 2010;56:661-9.

18. Narozny W, Kuczkowski J, Kot J, Stankiewicz C, Sicko Z, Mikaszewski B. Prognostic factors in sudden sensorineural hearing loss: our experience and a review of the literature. Ann Otol Rhinol Laryngol. 2006;115:553-8.

19. Atay G, Kayahan B, Çınar BÇ, Saraç S, Sennaroğlu L. Prognostic factors in sudden sensorineural hearing loss. Balkan Med J. 2016;33:87-93.

20. Haynes DS, O'Malley M, Cohen S, Watford K, Labadie RF. Intratympanic dexamethasone for sudden sensorineural hearing loss after failure of systemic therapy. Laryngoscope. 2007;117:3-15

21. Battista RA. Intratympanic dexamethasone for profound idiopathic sudden sensorineural hearing loss. Otolaryngol Head Neck $=$ Surg. 2005;132:902-5.

22. Lai $\mathrm{D}$, Zhao $\mathrm{F}$, Jalal $\mathrm{N}$, Zheng $\mathrm{Y}$. Intratympanic glucocorticosteroid therapy for idiopathic sudden hearing loss: meta-analysis of randomized controlled trials. Medicine (Balt). 2017;96:e8955. 